\title{
Does a Year Have 6 Months or 12? Implications for Delirium Detection Among Hospitalized Older General Medicine Patients
}

\author{
Wenxiao Zhou, $\mathrm{MS}^{7}$, Long H. Ngo, $\mathrm{PhD}^{1,2}$, Sharon K. Inouye, MD MPH' 2,3,4, Jacqueline Gallagher, $\mathrm{MS}^{7}$, \\ Erica K. Husser, $P h D^{5}$, Donna M. Fick, RN, $P h D^{5}$, and Edward R. Marcantonio, MD SM ${ }^{1,2,4}$ \\ 'Division of General Medicine, Department of Medicine, Beth Israel Deaconess Medical Center, Boston, MA, USA; ${ }^{2}$ Harvard Medical School, \\ Boston, MA, USA; ${ }^{3}$ Aging Brain Center, Institute for Aging Research, Hebrew SeniorLife, Boston, MA, USA; ${ }^{4}$ Division of Gerontology, Department of \\ Medicine, Beth Israel Deaconess Medical Center, Boston, MA, USA; ${ }^{5}$ Colleges of Nursing and Medicine, The Pennsylvania State University, University \\ Park, State College, PA, USA.
}

J Gen Intern Med 34(3):354-5

DOI: $10.1007 / \mathrm{s} 11606-018-4704-3$

(c) Society of General Internal Medicine 2018

\section{BACKGROUND}

Delirium is common, morbid, and costly, yet less than half of all cases are recognized clinically. ${ }^{1}$ Since inattention is a key feature of delirium, attention items are excellent screening tools for delirium. ${ }^{2}$ Months of the year backwards (MOYB) has been identified as the best single delirium screening item. ${ }^{2,3}$ Yet, there is significant variation in how MOYB is administered and scored. Some tools recommend asking the patient to recite all months from December to January, ${ }^{3}$ while others suggest reciting only from December to July. ${ }^{2,4}$

\section{OBJECTIVE}

Our aim was to evaluate these two MOYB administration strategies when MOYB is used as a single-item delirium screen, as part of a previously reported ultra-brief 2-item screen, ${ }^{3}$ and as part of the validated 3D-CAM diagnostic assessment. $^{5}$

\section{METHODS}

Data is from 201 general medicine patients $(\geq 75$ years old) enrolled in our 3D-CAM validation study. ${ }^{5}$ Participants were administered a detailed evaluation for delirium by a trained clinician applying DSM-4 criteria, and a researcher blinded to this evaluation administered the 3D-CAM. ${ }^{5}$ MOYB is one of 20 items in the 3D-CAM, and we recorded when participants made their first error

Wenxiao Zhou and Long H. Ngo contributed equally to this manuscript as first authors.

Donna M. Fick and Edward R. Marcantonio contributed equally as last authors.

Published online October 29, 2018 on this item. For the 12/full-MOYB (December to January) we defined "negative" (normal) as no error on any MOYB. For the 6/half MOYB (December to July) we defined "negative" (normal) as no error on any month, or the first error made after July (from June to January). We compared the 12/full to the 6/half MOYB under three scenarios-MOYB as a single screening item, as part of the 2-item screen, and as part of the full 3DCAM. For each scenario, comparing the screening and 3D-CAM results with the presence or absence of delirium from the clinical evaluation, we computed sensitivity, specificity, positive predictive value (PPV), and negative predictive value (NPV) with 95\% confidence intervals. Confidence intervals were computed using the exact binomial method. Differences in sensitivity and specificity were compared statistically using McNemar's test. We used SAS 9.4 for data analysis (SAS Institute, Cary, NC).

\section{FINDINGS}

The 201 participants had mean age (standard deviation) of 84 (5.5) years, $62 \%$ were women, $28 \%$ had dementia, and $21 \%$ had delirium based on the clinical evaluation. Across MOYB, $59.5 \%$ of first errors were made between December and July, while $40.5 \%$ were made between June and January. We found (Table 1) that administration of the 6/half MOYB reduces sensitivity in each scenario and has the most impact when MOYB is used as a single screening item. The 6/half MOYB improves specificity in the single-item and two-item screen scenarios, but not in the full 3D-CAM. Similarly, 6/half MOYB reduces NPV but improves PPV in the one and two-item screening scenarios, whereas it results in worse NPV and PPV in the 3D-CAM.

\section{DISCUSSION}

Administering MOYB from December-July vs. December-January substantially reduces sensitivity and NPV 
Table 1 Comparison of the Effect of Administering MOYB from December-July vs. December-January on Test Performance Relative to a Clinical Reference Standard Under 3 Scenarios

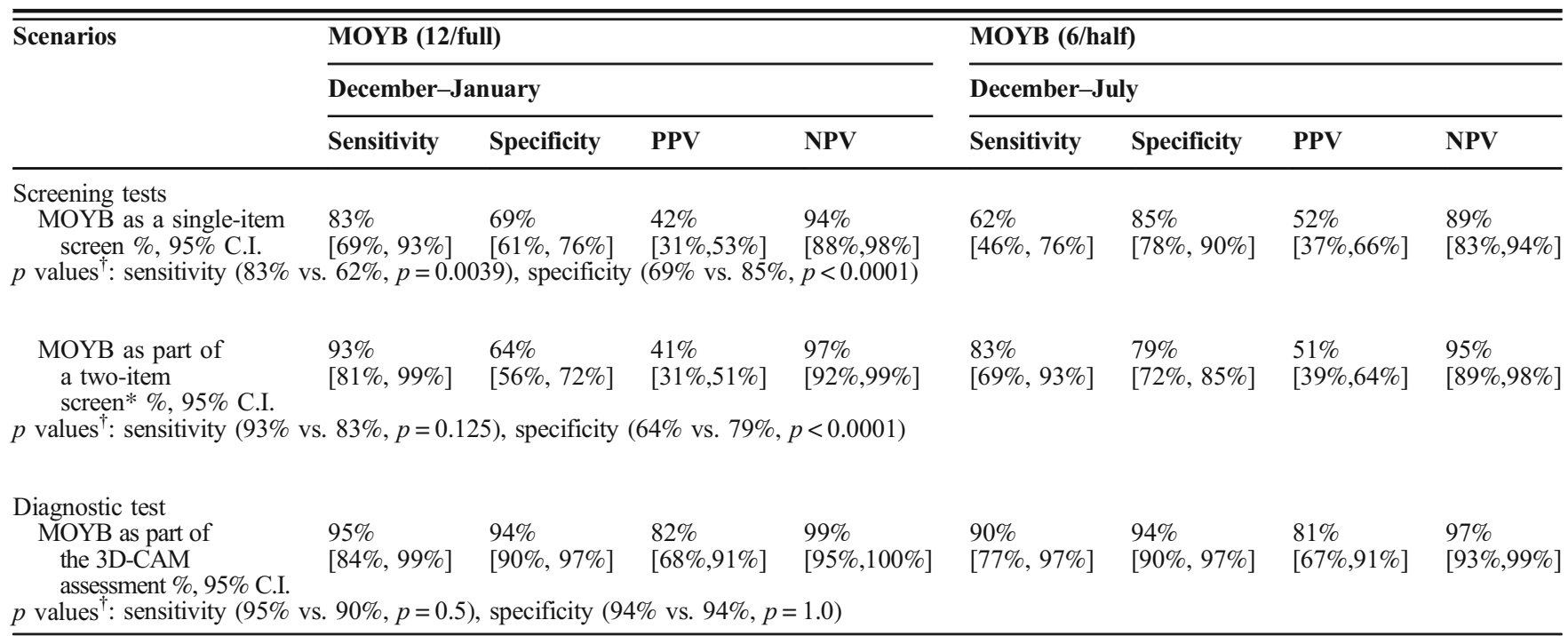

*The two-item screen consists of MOYB and "What is the day of the week?" Either item incorrect is considered a positive screen (reference 3)

${ }^{\dagger} P$ value from McNemar's test

PPV positive predictive value, NPV negative predictive value, MOYB months of the year backwards, C.I. confidence intervals

for delirium when MOYB is used alone or as part of a two-item screen. Notably, maximizing NPV is important in these ultra-brief screening scenarios, which have the goal of quickly ruling out delirium. "Negative" screens end the testing, whereas "positive" screens require further evaluation with a diagnostic test such as the 3D-CAM. In the full 3D-CAM, use of the 6/half MOYB results in reduced sensitivity and NPV with no improvement in specificity or PPV. Clinicians should carefully consider whether the time savings of administering MOYB only to July (in our simulations, only $10-15 \mathrm{~s})$ is worth the reduced detection rate for delirium.

Our study has some limitations. First, our results are based on secondary analysis of existing data; we did not prospectively test the two MOYB administration strategies in the clinical setting. Second, we only compared performance under three scenarios; the impact of using the 6/half MOYB on performance within other instruments may be different. Finally, our study was conducted in a very old population with a high prevalence of dementia. In younger populations, we would expect the 12/full MYOB to compare even more favorably relative to the $6 /$ half MYOB. This should be confirmed in a larger more representative sample, with inclusion of non-English speakers.

Based on our results, we believe that a year should indeed include all 12 months, particularly for maximizing detection of delirium.
Corresponding Author: Edward R. Marcantonio, MD SM; Division of General Medicine, Department of Medicine, Beth Israel Deaconess Medical Center, Boston, MA, USA (e-mail: emarcant@bidmc.harvard.edu).

Funding Sources This work was supported by the following grants: R01AG030618 (Marcantonio/Fick), K24AG035075 (Marcantonio), R24AG054259 (Inouye), and R01AG044518 (Inouye), all from the National Institute on Aging. The funding sources had no role in the drafting or submission of this manuscript.

\section{Compliance with Ethical Standards:}

Human Subjects: The Institutional Review Board of the Beth Israel Deaconess Medical Center approved all human subjects' research activities in this manuscript-IRB protocols 2008-P-000165 and 2015-P00096.

Conflict of Interest: The authors declare that they do not have a conflict of interest.

\section{REFERENCES}

1. Marcantonio ER. Delirium in Hospitalized Older Adults. N Engl J Med. 2017;377:1456-1466. Review.

2. O'Regan NA, Ryan DJ, Boland E, et al. Attention! A good bedside test for delirium? J Neurol Neurosurg Psychiatry. 2014;85:1122-1131.

3. Fick DM, Inouye SK, Guess J, et al. Preliminary development of an ultrabrief two-item bedside test for delirium. J Hosp Med. 2015; 10:645-650.

4. Han JH, Wilson A, Vasilevskis EE, et al. Diagnosing delirium in older emergency department patients: validity and reliability of the delirium triage screen and the brief confusion assessment method. Ann Emerg Med. 2013;62:457-465.

5. Marcantonio ER, Ngo LH, O'Connor M, et al. 3D-CAM: derivation and validation of a 3-minute diagnostic interview for CAM-defined delirium: a cross-sectional diagnostic test study. Ann Intern Med. 2014;161:554-561. 